\title{
Toxoplasmosis in Cancer Patients and Suggestion for Screening
}

\author{
Asian Pac J Cancer Prev, 20 (4), 985-986
}

\section{Dear Editor}

Dear Editor, we read the publication on "Toxoplasmosis an Overlooked Disease: Seroprevalence in Cancer Patients" with a great interest (Abdel Malek et al., 2018). Abdel Malek et al., (2018) "Cancer patients showed a significantly higher rate of infection with T. gondii. For that reason, we recommend the inclusion of a screening test for toxoplasmosis in their routine workup." It is interesting that this study use a less controls comparing to the studied cancer patients in seroprevalent rate study. Whether there is any selection bias is an interesting question. In fact, toxoplasmosis is an important infection and the asymptomatic seropositive rate is not uncommon in developing countries such as India (Mohan et al., 2002). Whether the cancer contribute to increase risk of getting toxoplamosis is an interesting question. In many reports, the high prevalent rate of seropositivity against toxoplasmosis are reported. Meta-analysis also showed that the cancer patients, especially for those who have immunosuppression status usually have seropositivity to toxoplasmosis (Jiang et al., 2015). However, an interesting concern is there has never been identified specific pathophysiological process on increased severity of toxoplasmosis in cancerous patients comparing to that seen in infection in normal non-cancer subjects. In endemic areas, such as tropical developing countries, seroprevalence of toxoplasmosis is high and the asymptomatic infection is common (Gohardehi et al., 2018). Due to the high seropsiotive asymptomatic infection, the routine screening for toxoplasmosis has never been suggested in the endemic developing countries. It is questionable on the exact usefulness of implementation of toxoplasmosis screening among cancer patients. This issue is interesting and should be further systematically investigate on advance and cost - utility.

\section{Conflict of interest}

None.

\section{References}

Abdel Malek R, Wassef R, et al (2018). Toxoplasmosis an overlooked disease: seroprevalence in cancer patients. Asian Pac J Cancer Prev, 27, 1987-91.

Gohardehi S, Sharif M, Sarvi S, et al (2018) The potential risk of toxoplasmosis for traffic accidents: A systematic review and meta-analysis. Exp Parasitol, 191, 19-24.

Jiang C, Li Z, Chen P, Chen L (2015). The seroprevalence of Toxoplasma gondii in Chinese population with cancer: A systematic review and meta-analysis. Medicine (Baltimore),
94, e2274.

Mohan B, Dubey ML, Malla N, Kumar R (2002). Seroepidemiological study of toxoplasmosis in different sections of population of Union Territory of Chandigarh. J Commun Dis, 34, 15-22.

\section{Beuy Joob $^{1 *}$, Viroj Wiwanitkit ${ }^{2}$}

${ }^{1}$ Sanitation 1 Medical Academic Center, Bangkok, Thailand, ${ }^{2}$ Honorary professor, dr DY Patil University, Pune, India.*For Correspondence: beuyjoob@hotmail.com

\section{Dear Editor}

Toxoplasmosis can result in serious complications in the immunocompromised patients as AIDS, cancer, and transplant patients. This is due to reactivation of a latent infection or due to acquisition of a new infection (Ahmadpour, 2014).

Are immunocompromised patients as cancer patients more prone to acquire the infection than immunocompetent individuals? This question has not been answered yet. As a first step towards finding an answer to this question we conducted our study published in your prestigious journal (Abdelmalek et al., 2018) aiming to determine if there is a difference in the prevalence rate between cancer patients and non-cancerous patients. Our study proved that there is a significant statistical difference between patients and controls. Further studies are on the go.

We considered four groups of patients, each contained 50 patients. So, the control group is similar in number to each group of patients. Still, the total number of patients is bigger than that of the control which is not uncommon in prevalence studies (many are referred to in our paper). This is acceptable as long as statistical analysis of the results are done.

Regarding the addressed question of the necessity of screening of cancer patients based on our results in developing countries; we are confident that prevention of serious complications of toxoplasmosis by performing a simple screening test that costs approximately 1 USD is cost beneficial especially that we found a high prevalence of infection in our study and we aim to highlight this to all clinicians especially in developing countries.

\section{References}

Ahmadpour E, Daryani A, Sharif M,et al (2014). Toxoplasmosis in immunocompromised patients in Iran: a systematic review and meta-analysis. J Infect Dev Ctries, 8, 1503-10.

Abdel Malek R, Wassef R, Rizk E,et al (2018). Toxoplasmosis Asian Pacific Journal of Cancer Prevention, Vol 19 
Beuy Joob and Viroj Wiwanitkit

an overlooked disease: Seroprevalence in cancer patients. Asian Pac J Cancer Prev, 19, 1987-91.

\section{Raafat Abdel Malek ${ }^{1 *}$, Rita Wassef ${ }^{2}$, Enas Rizk ${ }^{3}$, Hoda Sabry ${ }^{4}$, Nevine Tadros $^{4}$, Abdallah Boghdady ${ }^{3}$}

${ }^{1}$ Department of Clinical Oncology, ${ }^{3}$ Department of Medical Parasitology, Kasr Al-Ainy Faculty of Medicine, Cairo University, ${ }^{2}$ Department of Medical Parasitology, Faculty of Medicine, Helwan University, ${ }^{4}$ Department of Medical Parasitology, Theodor Bilharz Research Institute, Egypt. *For Correspondence: Raafat.malek@kasralainy.edu.eg 